\title{
¿Es el absceso hepático de origen hidatídico un factor de riesgo para el desarrollo de complicaciones postoperatorias en pacientes intervenidos por hidatidosis hepática?*
}

\author{
Drs. CARLOS MANTEROLA D. ${ }^{1,2}$, ANTONIO SANHUEZA C., ${ }^{2,3}$, \\ MANUEL VIAL G. ${ }^{1}$, JAVIER MORAGA C. ${ }^{1}$, GRUPO MINCIR \\ Departamento de Cirugía y Traumatología, Facultad de Medicina. \\ CIGES (Training, Investigation and Evidence for Based Health Medicine), Facultad de Medicina. \\ 3 Departamento de Matemáticas, Facultad de Ingeniería. \\ Universidad de La Frontera, Temuco, Chile.
}

\begin{abstract}
Liver abscess of hydatid origin as a risk factor for postoperative complications in hydatidosis
\end{abstract}

Background: Cyst infection and subsequent abscess formation is considered a complication of hepatic echinococcosis (HE). Aim: To determine whether liver abscess of hydatid origin (LAHO) is a risk factor for the development of postoperative complications (POC) in patients operated by HE. Material and Methods: Prospective cohort study. Patients consecutively operated by HE between 2000 and 2007 were studied. LAHO was considered as exposure and POC as outcome. Results: Fifty two patients with LAHO, aged $50 \pm 21$ years (52\% females) and 126 without LAHO, aged $48 \pm 20$ years (56\% females) were studied. Patients with LAHO had a higher frequency of coexistent cyst lesions and a lower frequency of biliary communications. The frequency of complications was 28.9 and $11.1 \%$ among patients with and without LAHO, respectively ( $\mathrm{p}<0.01$, crude risk ratio of $2.6,95 \%$ confidence intervals $1.4-5.0)$. Logistic regression showed a significant interaction of alkaline phosphatases with risk calculation. The risk ratio, adjusting by this variable, changed to 2.45 (95\% confidence intervals 1.3-5.0). Conclusions: LAHO is a risk factor for the development of POC in patients operated by HE.

Key words: Echinococcosis, hepatic, hepatic hydatid cyst, liver abscess.

\section{Resumen}

Introducción: La infección del quiste y subsiguiente formación de un absceso hepático de origen hidatídico (AHH) constituye una complicación evolutiva de la hidatidosis hepática (HH). Objetivo: Determinar si el AHH constituye un factor de riesgo (FR) para el desarrollo de complicaciones postoperatorias

\footnotetext{
*Recibido el 31 de Marzo de 2009 y aceptado para publicación el 15 de Abril de 2009.

Financiado parcialmente por la Dirección de investigación de la Universidad de La Frontera.

Correspondencia: Dr. Carlos Manterola D.

Manuel Montt 112, Oficina 408. Temuco, Chile.

E-mail: cmantero@ufro.cl
} 
(CPO) en pacientes intervenidos por HH. Material y Método: Estudio de cohorte prospectiva. Se estudiaron pacientes intervenidos consecutivamente entre 2000 y 2007. La variable de exposición fue tener AHH y la variable resultado, el desarrollo de CPO (considerada de forma dicotómica). El tamaño de la muestra se calculó considerando nivel de significación de 5\%, potencia de $80 \%$, riesgo de $\mathrm{CPO}$ en pacientes con $\mathrm{AHH}$ de 24,4\% y sin AHH de 6,5\%; relación paciente con AHH: sin AHH de 1: 2; lo que determinó una muestra de 52 pacientes con AHH y 104 sin AHH. Se aplicaron análisis bivariados paramétricos y no paramétricos. Se calculó incidencia de CPO, riesgo relativo (RR) e intervalos de confianza del 95\% (IC 95\%); y se controló el efecto de variables clínicas, de laboratorio, evolutivas del parásito y quirúrgicas, aplicando modelos de regresión logística. Resultados: Las cohortes se conformaron por 52 pacientes con AHH y 126 sin AHH, con una mediana de edad de 45 años (59\% de género femenino). La distribución de las variables categóricas fue similar en las cohortes en estudio, a excepción de "lesiones quísticas coexistentes" y "comunicaciones biliares". La incidencia de morbilidad fue $28,9 \%$ para la cohorte con AHH, y de $11,1 \%$ para la cohorte sin AHH ( $\mathrm{p}=0,0036$; RR crudo de 2,6 [IC 95\% 1,4 - 5,0]). El modelo de regresión logística permitió constatar que la variable "fosfatasa alcalina", interactuaba en la asociación en estudio $(p=0,018)$, razón por la que se controló por ésta, obteniéndose un RR ajustado de 2,45 con IC 95\% de 1,3-5,0. Conclusión: Se verificó que el AHH constituye un FR para el desarrollo de CPO en pacientes intervenidos por HH.

Palabras clave: Echinococcosis hepática, quiste hidatídico hepático, hidatidosis hepática, absceso hepático, absceso hepático de origen hidatídico, morbilidad, factores de riesgo.

\section{Introducción}

Los resultados de la cirugía de la hidatidosis hepática $(\mathrm{HH})$ persisten como tema relevante de discusión, puesto que a pesar de las avances científicos y tecnológicos, el desarrollo de complicaciones postoperatorias (CPO), sigue siendo elevado según los distintos reportes; desde cifras muy altas $(57 \% \text { y hasta } 68,5 \%)^{1-4}$; hasta otras que parecen ser más reales, entre $7 \%$ y $18 \%$, cuando se consideran pacientes con $\mathrm{HH}$ no complicada ${ }^{5,6}$.

Por otro lado, existe evidencia que sugiere que algunas complicaciones evolutivas de la $\mathrm{HH}$ como la infección del quiste y la subsiguiente formación de un absceso, se asocian a mayor prevalencia de $\mathrm{CPO}^{1,6-8}$. Esta entidad que hemos denominado "absceso hepático de origen hidatídico" (AHH) $)^{9,10}$, es de diagnóstico difícil y pronóstico incierto; y a pesar de ello, existen escasos reportes referentes al tema, tanto a nivel nacional como internacional. La mayor parte de las veces los casos de $\mathrm{AHH}$, son incorporados como parte de una serie de casos que se publica, por lo que los resultados de tratamientos y pronóstico se confunden con pacientes con $\mathrm{HH}$ no complicada.

En 1997, reportamos los primeros resultados de un enfoque quirúrgico de pacientes con $\mathrm{AHH}^{9}$ intervenidos como casos de urgencia entre 1994 y 1996; posicionando la tesis que el simple drenaje quirúrgico se asociaba con curso postoperatorio poco satisfactorio, que hacía necesario la realización de procedimientos quirúrgicos complementarios con posterioridad. Luego, en 2003, publicamos una nueva serie de casos de pacientes con $\mathrm{AHH}$ intervenidos quirúrgicamente de forma consecuti- va, en los que propusimos la realización del tratamiento definitivo del problema (drenaje de la colección, aseo de la cavidad y tratamiento del parásito mediante periquistectomía total o subtotal). Con esta estrategia terapéutica obtuvimos una mediana de estancia hospitalaria de 5 días y, con una mediana de seguimiento de 32 meses, constatamos una morbilidad de $24,4 \%$, una mortalidad de $2 \%$ y ausencia de recurrencia de la enfermedad ${ }^{10}$.

Basada en toda la experiencia antes relatada en relación al desarrollo de $\mathrm{CPO}$, surgió la hipótesis que dió origen a este estudio que es que el AHH constituye un factor de riesgo (FR) para el desarrollo de CPO en pacientes intervenidos quirúrgicamente por $\mathrm{HH}$.

Por ende, el objetivo de este estudio es determinar si el AHH constituye un FR para el desarrollo de $\mathrm{CPO}$ en pacientes intervenidos por $\mathrm{HH}$.

\section{Material y Método}

\section{Diseño del estudio: Cohorte concurrente o prospectiva.}

Criterios de inclusión: Se incluyeron pacientes intervenidos de forma consecutiva por $\mathrm{HH}$ en los Servicios de Cirugía y Urgencias del Hospital Hernán Henríquez Aravena de Temuco, desde 2000 y 2007, por el primer autor (CM); lo que representa una muestra no probabilística de casos consecutivos. Se excluyeron pacientes intervenidos por $\mathrm{HH}$ vía laparoscópica. Se tuvo seguimiento de los sujetos, desde el momento de la cirugía hasta el momento del cierre del estudio; a través de controles clínicos, de laboratorio y ecotomográficos seriados 
en los meses $1,6,12,24,36,48,60,72$ y 84 . El período de reclutamiento se extendió entre enero de 2000 y junio de 2007; y el período se seguimiento concluyó en junio de 2008.

Variables de interés: La variable de exposición fue tener un $\mathrm{AHH}$; y la variable resultado, el desarrollo de CPO (considerada en forma dicotómica, con un seguimiento mínimo de 12 meses). Las variables de control fueron clínicas (edad, género; enfermedades coexistentes y antecedentes de cirugías previas por $\mathrm{HH}$ [ambas dicotomizadas en sí y no]); de laboratorio (hematocrito, recuento total de leucocitos, bilirrubina total, fosfatasa alcalina y transaminasas); evolutivas y propias del parásito (número de quistes [dicotomizada en uno y dos o más], diámetro del quiste, comunicaciones biliares [categorizada en ninguna, una y dos o más] y coexistencia de otras lesiones hidatídicas [dicotomizada en sí y no]); y quirúrgicas (tipo de cirugía [categorizada en quistectomía subtotal, periquistectomía y resección hepática], tratamiento de la cavidad residual [categorizada en epiploplastia y capitonaje y ningún tratamiento] y otras cirugías realizadas en forma simultánea a la cirugía de la $\mathrm{HH}$ [dicotomizada en sí y no]). Se controló por las variables antes mencionadas de forma tal de determinar un eventual efecto de confusión o de modificación del efecto que pudiesen tener éstas en la asociación en estudio.

Tamaño de la muestra: La estimación del tamaño de la muestra se calculó considerando un nivel de significación de $5 \%$, potencia de $80 \%$, riesgo de desarrollo de $\mathrm{CPO}$ en pacientes con $\mathrm{AHH}$ de $24,4 \%$ y $\sin \mathrm{AHH}$ de 6,5\%; relación paciente con $\mathrm{AHH}$ y sin $\mathrm{AHH}$ de 1:2; lo que determinó una muestra mínima de 52 pacientes con AHH y 104 sin AHH (es decir, un total de 156 sujetos a estudio).

Plan de análisis: Utilizando los paquetes estadísticos Epi-info 6.0 y Stata SE 9.0, se realizó un análisis exploratorio de los datos y posteriormente se aplicó estadística descriptiva con cálculo de promedios y desviaciones estándar, medianas y valores extremos para variables contínuas; y, cálculo de porcentajes para variables categóricas. Luego, se aplicó estadística analítica, utilizando t-test, análisis de la varianza (ANOVA) y métodos no paramétricos para variables contínuas; $\chi^{2}$ de Pearson y exacto de Fisher para variables categóricas. Posteriormente, se calculó la incidencia o riesgo absoluto (RA) de desarrollo de CPO para cada cohorte y el riesgo relativo (RR) crudo de la asociación en estudio, de modo tal de determinar la magnitud del efecto. Finalmente, se aplicaron modelos de regresión logística, para ajustar por las variables de control; calculando el RR ajustado y la precisión de la medición a través del cálculo de los respectivos intervalos de confianza del 95\% (IC 95\%) del RR calculado.

Aspectos éticos: Se observaron los principios Helsinki. Además, la confidencialidad de los datos de cada paciente fue asegurada a través de la codificación de cada una de las variables analizadas.

\section{Resultados}

Las cohortes se compusieron por 52 pacientes con AHH y 126 sin $\mathrm{AHH}$; es decir, un total de 178 sujetos a estudio, con una mediana de edad de 45 años y $59 \%$ de género femenino. La mediana de seguimiento de ambas cohortes fue de 39 meses (12 a 84 meses).

Al comparar las variables contínuas entre los grupos en estudio, se verificaron diferencias estadísticamente significativas en las variables edad, recuento total de leucocitos y determinación de fosfatasas alcalinas; en todas las cuales se registraron valores superiores en el grupo de pacientes con AHH (Tabla 1).

Tabla 1. Comparación de variables contínuas en las cohortes en estudio

\begin{tabular}{lccc}
\hline Variable & $\begin{array}{c}\text { Con } \mathbf{A H H} \\
(\mathbf{n}=\mathbf{5 2})\end{array}$ & $\begin{array}{c}\text { Sin } \mathbf{A H H} \\
(\mathbf{n}=\mathbf{1 2 6})\end{array}$ & $\mathbf{p}$ \\
\hline Edad (años) & $49,9 \pm 20,9$ & $47,8 \pm 19,8$ & 0,0047 \\
Leucocitos (x mm $)$ & $13.223 \pm 3.125$ & $8.499 \pm 3.125$ & 0,0001 \\
Bilirrubina (mg/dL) & $2,2 \pm 4,3$ & $1,2 \pm 1,9$ & 0,1723 \\
F. Alcalina (U/L) & $767,4 \pm 605,8$ & $490,3 \pm 470,7$ & 0,0068 \\
ASAT (U/L) & $62,8 \pm 76,7$ & $52,9 \pm 96,2$ & 0,5888 \\
ALAT (U/L) & $60,9 \pm 69,3$ & $57,7 \pm 103,9$ & 0,8259 \\
Diámetro quiste (cm) & $16,1 \pm 7,2$ & $14,2 \pm 6,6$ & 0,1418 \\
Hospitalización (días) & $6,9 \pm 4,3$ & $5,8 \pm 3,9$ & 0,0850 \\
\hline
\end{tabular}


Tabla 2. Comparación de variables dicotómicas en las cohortes en estudio

\begin{tabular}{|c|c|c|c|}
\hline Variable & $\begin{array}{c}\text { Con AHH } \\
(n=52)\end{array}$ & $\begin{array}{l}\text { Sin AHH } \\
(n=126)\end{array}$ & p \\
\hline \multicolumn{4}{|l|}{ Género (\%) } \\
\hline Femenino & 51,5 & 56,2 & 0,619 \\
\hline Masculino & 48,5 & 43,8 & \\
\hline \multicolumn{4}{|c|}{ Cirugías previas por HH (\%) } \\
\hline No & 90,3 & 89,7 & 1,0000 \\
\hline Sí & 9,6 & 10,3 & \\
\hline \multicolumn{4}{|c|}{ Número quistes (\%) } \\
\hline Uno & 57,6 & 65,7 & 0,374 \\
\hline Dos o más & 42,4 & 34,3 & \\
\hline \multicolumn{4}{|c|}{ Enfermedades coexistentes (\%) } \\
\hline No & 90,4 & 90,5 & 1,0000 \\
\hline Sí & 9,6 & 9,5 & \\
\hline \multicolumn{4}{|c|}{ Lesiones quísticas coexistentes (\%) } \\
\hline No & 82,7 & 66,4 & 0,0137 \\
\hline Sí & 17,3 & 33,6 & \\
\hline \multicolumn{4}{|c|}{ Otra cirugía realizada (\%) } \\
\hline No & 15,4 & 26,9 & 0,0720 \\
\hline Sí & 84,6 & 73,1 & \\
\hline
\end{tabular}

Al comparar las variables dicotómicas entre los grupos en estudio, se comprobó diferencias estadísticamente significativas sólo en la variable "lesiones hidatídicas coexistentes", las que fueron más frecuentes en el subgrupo de sujetos sin $\mathrm{AHH}$ (33,6\% vs $17,3 \% ; \mathrm{p}=0,0137)$. Tabla 2 .

Al comparar las variables categóricas entre los grupos en estudio, se constató diferencias estadísticamente significativas sólo en la variable "presencia de comunicaciones biliares", las que fueron más frecuentes en el subgrupo de sujetos con $\mathrm{AHH}$ (76,9\% vs 57,1\%; $\mathrm{p}=0,0061)$. Tabla 3 .

De este modo, la incidencia o RA de CPO para la cohorte con AHH fue de 15/52, es decir 0,289 $(28,9 \%)$; y la incidencia o RA de CPO para la sin AHH fue de 14/126, es decir 0,111 (11,1\%); con un valor de $p=0,0036$ y un RR crudo de 2,6 [IC 95\% de 1,4 - 5,0]). Las causas más frecuentes de CPO fueron las de origen respiratorio (12 casos; 6,7\%), seguidas por la infección de sitio operatorio $(7 \mathrm{ca}-$ sos; $3,9 \%$ ). Tabla 4.

Sin embargo, como 5 variables se asociaron en forma significativa en los análisis bivariados (edad, recuento total de leucocitos, determinación fosfatasas alcalinas, lesiones hidatídicas coexistentes y existencia de comunicaciones biliares); se realizó un ajuste aplicándose modelos de regresión logís-
Tabla 3. Comparación de variables categóricas en las cohortes en estudio

\begin{tabular}{|c|c|c|c|}
\hline Variable & $\begin{array}{c}\text { Con AHH } \\
(n=52)\end{array}$ & $\begin{array}{l}\text { Sin AHH } \\
(n=126)\end{array}$ & $\mathbf{p}$ \\
\hline \multicolumn{4}{|c|}{ Comunicaciones biliares (\%) } \\
\hline Ninguna & 23,1 & 42,9 & 0,0061 \\
\hline Una & 57,7 & 32,5 & \\
\hline Dos o más & 19,2 & 24,6 & \\
\hline \multicolumn{4}{|c|}{ Cirugía realizada en quiste (\%) } \\
\hline Quistectomía subtotal & 77,4 & 81,8 & 0,795 \\
\hline Periquistectomía & 11,9 & 12,1 & \\
\hline Lobectomía & 10,7 & 6,1 & \\
\hline \multicolumn{4}{|c|}{ Tratamiento cavidad residual (\%) } \\
\hline Epiploplastía & 48,5 & 42,4 & 0,150 \\
\hline Capitonaje & 27,8 & 18,2 & \\
\hline Ninguno & 23,7 & 39,4 & \\
\hline
\end{tabular}

tica, en los que se determinó que la variable "fosfatasa alcalina", interactuaba en la asociación $(\mathrm{p}=0,018)$, razón por la que se controló por ésta, obteniéndose un RR ajustado de 2,45; con IC de $95 \%$ de $1,3-5,0$.

Por otra parte, no se registró recurrencia en la cohorte con AHH y un caso en la cohorte sin $\mathrm{AHH}$ $(0,8 \%)$; la que se diagnosticó 36 meses después de la cirugía inicial.

Por otra parte, se registró mortalidad en un paciente del grupo con AHH (1,9\%) y ausencia de mortalidad en el grupo de pacientes sin AHH $(0,0 \%)$.

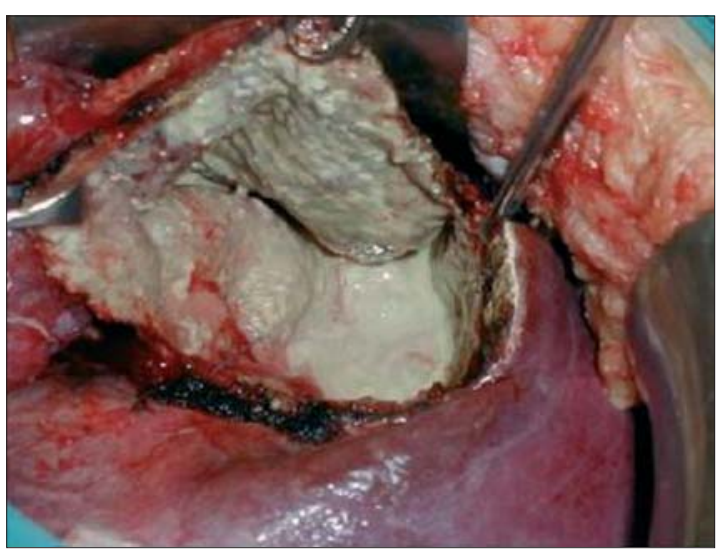

Figura 1. Imagen intraoperatoria, correspondiente a uno de los pacientes con AHH. Se trata de una lesión de 15 $\mathrm{cm}$ de diámetro, localizada entre los segmantos VII y VIII; en la que finalmente se practicó una quistectomía subtotal asociada a omentolpastia para el tratamiento de la cavidad residual. 
Tabla 4. Etiología de las CPO en las cohortes en estudio

\begin{tabular}{llcrr}
\hline Variable & & $\begin{array}{c}\text { Con AHH } \\
(\mathbf{n}=\mathbf{5 2})\end{array}$ & $\begin{array}{c}\text { Sin AHH } \\
(\mathbf{n}=\mathbf{1 2 6})\end{array}$ & $\begin{array}{c}\text { Total } \\
(\mathbf{n}=\mathbf{1 7 8})\end{array}$ \\
\hline Complicaciones respiratorias & $\left(\mathrm{N}^{\circ}\right.$ casos $\left.-\%\right) *$ & $7-13,5$ & $5-3,9$ & $12-6,7$ \\
Infección del sitio operatorio & $\left(\mathrm{N}^{\circ} \operatorname{casos}-\%\right)$ & $4-7,7$ & $3-2,4$ & $7-3,9$ \\
Infección del tracto urinario & $\left(\mathrm{N}^{\circ} \operatorname{casos}-\%\right)$ & $2-3,8$ & $2-1,6$ & $4-2,3$ \\
Hernia incisional & $\left(\mathrm{N}^{\circ} \operatorname{casos}-\%\right) * *$ & $1-1,9$ & $3-2,4$ & $4-2,3$ \\
Fístula biliar & $\left(\mathrm{N}^{\circ}\right.$ casos $\left.-\%\right) * * *$ & $1-1,9$ & $1-0,8$ & $2-1,1$ \\
Total de CPO & $\left(\mathrm{N}^{\circ} \operatorname{casos}-\%\right)$ & $15-28,8$ & $14-11,1$ & $29-16,3$ \\
\hline
\end{tabular}

*Las complicaciones respiratorias se resumen en 7 atelectasias basales ( 3 casos en la cohorte con AHH y 4 casos en la cohorte sin $\mathrm{AHH}$ ), que se resolvieron con ejercicios kinésicos; y 5 neumonías basales (4 casos en la cohorte con AHH y 1 en la cohorte sin $\mathrm{AHH}$ ), todos ellos requirieron de tratamientos con esquemas antibióticos para neumonía intrahospitalaria. **Los 4 pacientes que desarrollaron esta complicación fueron reintervenidos para la corrección de su eventración aplicando una placa de polipropileno. El diagnóstico de esta complicación se verificó al sexto mes en el paciente con AHH; y entre los 12 y 24 meses en los pacientes sin $\mathrm{AHH} .{ }^{* * *}$ Se trató de fístulas que se manifestaron clínicamente alrededor del tubo de Kehr, pero que cedieron de forma espontánea antes de los 15 días del postoperatorio sin requerir para ello de algún procedimiento accesorio.

El caso de mortalidad se trató de una paciente que presentó una insuficiencia respiratoria aguda de curso fulminante en 36 horas, que fue atribuida a un tromboembolismo pulmonar masivo.

\section{Discusión}

Como se mencionó en la introducción, existen escasos reportes referentes a $\mathrm{AHH}^{9-18}$. Incluso utilizando estrategias de búsqueda sensibles, con términos $\mathrm{MeSH}$, palabras libres y operadores booleanos; sólo aparecen los estudios antes señalados, la mayor parte de los cuales se trata de series de casos pequeñas (6 a 72 pacientes), varias de carácter retrospectivo, algunas de las cuales corresponden a publicaciones antiguas, que aportan un nivel de evidencia tipo $4^{19}$. Además, de algunas de ellas sólo se puede obtener el resumen ${ }^{12,13,17}$, y de una, sólo se logra encontrar la referencia ${ }^{12}$. En términos generales, de ellas se logra obtener información heterogénea y confusa, pues hay mezcla de pacientes con $\mathrm{AHH}$, absceso hepático piógeno y amebiano. Los artículos aportan datos referentes al cuadro clínico, el apoyo de las imágenes en el proceso diagnóstico, la descripción de estrategias terapéuticas (que van desde la cirugía y sus diversas opciones técnicas, hasta el drenaje percutáneo y endoscópico), y los resultados obtenidos en términos de morbilidad y mortalidad (no reportada por todos ellos), pero que fluctúa en cifras cercanas al $30 \%$ y $10 \%$ respectivamente.

Por otra parte, este es un estudio diseñado para valorar pronóstico y que se realizó utilizando un diseño apropiado para tal efecto; es decir, un estudio de cohorte concurrente o prospectiva, que aporta un nivel de evidencia tipo $1 \mathrm{~b}^{19}$; $\mathrm{y}$ hasta donde conocemos es el único referente a este tema, en el que se ha utilizado este tipo de diseño.

En este estudio se verificó que el presentar un $\mathrm{AHH}$ es un FR para el desarrollo de CPO en pacientes intervenidos por $\mathrm{HH}$; en términos que la probabilidad de desarrollar CPO es 2,45 veces mayor en presencia de un $\mathrm{AHH}$ respecto de pacientes que se interviene por $\mathrm{HH}$ sin $\mathrm{AHH}$; esto, con un adecuado nivel de precisión, que es aportado por los IC 95\% de la estimación; y que en este caso fueron estrechos $(1,3-5,0)$ y superiores a 1 en sus valores mínimo y máximo. Este antecedente nos otorga mayor información al momento de la toma de decisiones en pacientes que se intervienen por $\mathrm{HH}$; debido a que la mayor parte de las CPO son de tipo séptico, tanto a nivel local como sistémico.

Aún así, somos concientes que el estudio presenta potenciales fuentes de sesgos que merecen ser comentadas. En la etapa de reclutamiento y seguimiento, los sesgos fueron minimizados con un seguimiento completo de las cohortes, un enmascaramiento de los informes y la recolección de los datos de los sujetos en estudio, los que se trabajaron en forma de códigos que no se develaron hasta el momento del análisis. Y en la etapa de análisis de los datos se planificó una justificación por potenciales variables de confusión y modificadoras de efecto, a partir de las variables de control.

Otra consideración que merece ser comentada 
tiene que ver con la estimación del tamaño de la muestra, la que se calculó con base a información previa que, como se comentó anteriormente, puede ser poco válida debido a la calidad metodológica de los estudios que la generaron; razón que permite comentar que en el análisis de algunas variables ("tiempo de hospitalización" y "otra cirugía realizada") se observó una tendencia a la significación estadística; la que puede estar dada por una muestra insuficiente de pacientes en los grupos en estudio, induciendo a un potencial error tipo $\alpha$, es decir asumiendo que no existe una diferencia estadísticamente significativa cuando en realidad existe.

Finalmente, vale la pena comentar la ausencia de recurrencia de $\mathrm{HH}$ en la cohorte con $\mathrm{AHH}$. Hecho que puede ser explicado por un estudio anteriormente publicado por nuestro grupo en el que se demostró la ausencia de protoescólices vivos al interior de los quistes en pacientes con $\mathrm{AHH}^{20}$.

\section{Referencias}

1. Sapunar J, Rappoport J, Sapunar J, Cumsille F. Quiste hidatídico hepático: características clínicas, factores pronósticos y resultados quirúrgicos. Parasitol al Día 1989; 13: 52-63.

2. Pinto P. Hidatidosis hepática: estudio de una serie de 534 casos. Rev Chil Cir 1991; 43: 184-187.

3. Camacho J, Reyes J, Pérez A. Cirugía del quiste hidatídico hepático. Rev Chil Cir 1996; 48: 479-482.

4. Correa S, Culqui C, Pinto M. Hidatidosis hepática: revisión de casos intervenidos quirúrgicamente en el Hospital Militar Central Lima 1990-1999. Rev Gastroenterol Perú 2000; 20: 261-269.

5. Manterola C, Molina E, Fernández O, Garrido L, Acencio L, Barroso M. Quistectomía subtotal. Una alternativa quirúrgica racional en el tratamiento de la hidatidosis hepática. Rev Chil Cir 1998; 50: 621-629.

6. Manterola C, Vial M, Pineda V, Sanhueza A, Barroso M. Factors associated with morbidity in liver hydatid surgery. ANZ J Surg 2005; 75: 889-892.

7. Manterola C, Acencio L, Bahamondes JC, Barroso M. Hidatidosis hepática: Estudio descriptivo de algunos aspectos clínicos y terapéuticos. Rev Chil Cir 1997; 49: $352-359$.
8. Kayaalp C, Bzeizi K, Demirbag AE, Akoglu M. Biliary complications after hydatid liver surgery: incidence and risk factors. J Gastrointest Surg 2002; 6: 706712.

9. Manterola C, Acencio L, Garrido L, Molina E, Barroso M. Absceso hepático de origen hidatídico. Resultados de la cirugía de urgencia. Rev Chil Cir 1997; 49: 510-514.

10. Manterola C, Barroso M, Vial M, Bustos L, Muñoz S, Losada $\mathrm{H}$, et al. Liver abscess of hydatid origin: clinical features and results of aggressive treatment. ANZ J Surg 2003; 73: 220-224.

11. Payer SJ, Amengual JM, Bobadilla M, Revilla JM, Barrera R, Lozano R. Abscesos piógenos hepáticos con fístula hepatobronquial. A propósito de dos casos, uno de ellos curado por vómica. Cir Esp 1980; 4: $264-$ 272 .

12. Stoianov G, Derezhian KH. Diagnosis and treatment of suppurative echinococcal cysts of the liver. Khirurgiia (Sofiia) 1981; 34: 530-535.

13. Ordabekov SO. Suppurative echinococcal cysts in the abdominal cavity. Vestn Khir Im I I Grek 1985; 135 : 58-62.

14. Karavias D, Panagopoulos C, Vagianos C, Vagenas C, Rathosis S, Androulakis J. Infected echinococcal cyst. A common cause of pyogenic hepatic abscess. Ups J Med Sci 1988; 93: 289-296.

15. Mayer DA, Fried B. Aspects of human parasites in which surgical intervention may be important. Adv Parasitol 2002; 51: 1-94.

16. Sharma BC, Agarwal N, Garg S, Kumar R, Sarin SK. Endoscopic management of liver abscesses and cysts that communicate with intrahepatic bile ducts. Endoscopy 2006; 38: 249-253.

17. Ga-batov SP, Ga-batova DS. Clinical finding and treatment of festered hepatic echinococcosis. Khirurgiia (Mosk) 2006; 6: 16-18.

18. Das K, Sakuja P, Aggarwal A, Puri AS, Tatke M. Non-resolving liver abscess with Echinococcus crossreactivity in a non-endemic region. Indian J Gastroenterol 2007; 26: 92-93.

19. http://www.cebm.net/index.aspx?o=1025. Accedido el 13 de marzo de 2009.

20. Manterola C, Vial M, Melo A, Oberg C, Fonseca F. Viability and fertility of human hepatic hydatid cysts. World J Surg 2006; 30: 227-232. 